\title{
Métodos para Avaliação Ergonômica em Calçados
}

\author{
Methods for Ergonomic Footwear Evaluation
}

ANTUNES, Ana Cláudia; Mestranda em Design; Universidade do Estado de Santa Catarina (UDESC) anacantunes@outlook.com.br

SANTOS, Flavio Anthero Nunes Vianna dos; Doutor em Engenharia de Produção; Universidade do Estado de Santa Catarina (UDESC)

flavioanvs@hotmail.com

REIS, Alexandre Amorim dos; Doutor em Engenharia de Produção; Universidade do Estado de Santa Catarina (UDESC)

alexandre.reis@pq.cnpq.br

\section{Resumo}

O presente artigo tem como objetivo identificar, organizar e sintetizar os principais métodos utilizados recentemente para análise ergonômica em diferentes tipos de calçados. Utilizou-se uma revisão bibliográfica sistemática para selecionar os trabalhos analisados. O intuito é poder contribuir e orientar futuras pesquisas relacionadas à fatores humanos em calçados, objetivando colaborar para o aumento da condição de conforto no uso do artefato.

Palavras Chave: métodos; ergonomia; calçados.

\section{Abstract}

This article aims to identify, organize and synthesize the main methods recently used for ergonomic analysis in different types of footwear. A systematic bibliographic review was used to select the papers analyzed. The aim is to be able to contribute and guide future research related to human factors in shoes, aiming to collaborate to increase the comfort condition in the use of the artifact.

Keywords: methods; ergonomics; footwear. 


\section{Introdução}

Dentro do design, a ergonomia busca melhorar a interação entre usuários e produtos, focando no bem-estar e satisfação das pessoas. Os fatores humanos têm como objetivo melhorar a vida de um maior número de pessoas, promovendo a inclusão e a qualidade de vida de todos. A ergonomia é definida então por Gomes Filho (2010) como uma ciência com finalidade de sempre buscar a melhor adaptação do objeto aos seres vivos, sendo um dos enfoques, a eficácia de uso ou de operacionalidade dos produtos e sistemas.

O processo de design deve ser sistemático e organizado visando sempre resultados. Para tal fim se faz necessário o uso de métodos de projeto, ferramentas e técnicas de exploração do processo criativo. A metodologia é área que engloba a teoria do conhecimento sobre métodos, ferramentas e técnicas.

De acordo com Santos (2017) os métodos são um conjunto de operações necessárias para a realização de um projeto ou pesquisa, dispostas de forma lógica e coerente, e facilitam na organização dos problemas solucionando-os em partes com mais eficiência, rapidez e segurança. É fundamental definir o que se deseja pesquisar para então selecionar o tipo de método mais adequado ou qual a melhor combinação de métodos.

Considerando uma maior preocupação atual com os fatores humanos nos mais variados produtos de interação direta com os usuários, muitas são as pesquisas voltadas para os artefatos de uso cotidiano como é o caso de peças de vestuário, acessórios e calçados. Apesar dos avanços ergonômicos e tecnológicos, ainda é possível perceber dificuldades de interação com alguns produtos.

Ao longo da história, os calçados tiveram a função inicial de proteger os pés. Porém com a evolução da civilização, o mesmo foi adquirindo outros atributos, passando a ter um papel importante na distinção entre as classes sociais e ganhando paralelamente um forte apelo estético.

Os calçados, cujo objetivo inicial é proporcionar proteção e conforto, podem tanto quanto causar lesões e doenças. Muitas pessoas sentem desconforto no uso deste artefato, apesar da grande variedade de modelos disponível no mercado. $O$ uso de sapatos inadequados pode causar não apenas problemas ortopédicos, mas também de pele e unhas.

Observando-se esse cenário o presente trabalho objetiva identificar, organizar e sintetizar quais os principais métodos utilizados recentemente para análise ergonômica em diferentes tipos de calçados.

\section{Ergonomia no calçado}

A principal função do calçado é proteger os pés e prevenir lesões. Apesar do apelo estético adquirido ao longo dos anos pelo produto, é de extrema importância que seja confortável. 0 estudo da ergonomia no calçado pretende tornar a interação entre o pé e o artefato em uma relação harmoniosa, proporcionando bom calce e marcha mais agradável.

Choklat (2012) afirma que o sapato é composto de diversas partes e, apesar de normalmente serem fabricadas independentemente, precisam atuar em conjunto, de forma dinâmica. De acordo com Cipatex apud Anselmo (2014) o calçado é dividido em duas partes principais, sendo a construção superior chamada de cabedal e a inferior de solado, cada uma delas 
é constituída por uma série de componentes com características e funções específicas variando de acordo como modelo e processo de fabricação.

A confecção de um sapato começa a partir de uma forma, que segundo Choklat (2014) é um molde em formato de pé usado para montar o sapato, porém a forma não deve ter o formato e as medidas exatas do pé. Ela é projetada incluindo as medidas das folgas que devem existir entre o pé o calçado, para permitir a movimentação. Ainda segundo o autor a forma deve ser projetada de modo a acomodar o salto e sola do sapato.

Bozano e Oliveira (2008) alertam que para a fabricação de calçados verdadeiramente confortáveis são indispensáveis conhecimentos sobre anatomia e fisiologia do pé, normas de conforto, materiais e engenharia de produção, além de obter conhecimentos sobre o modo que o consumidor irá usá-lo, já que o uso contínuo de um mesmo calçado pode interferir na construção e materiais do mesmo.

De acordo com Anselmo (2014) os fatores humanos nos calçados são estudados com foco na antropometria, biomecânica e usabilidade, a análise de cada um destes fatores é extremamente importante para que o calçado seja construído de forma adequada e possa atender as necessidades do usuário proporcionando conforto em todo o seu contexto.

Segundo Choklat (2012) é importante entender tanto a anatomia básica do pé quanto a constituição interna do sapato. O pé humano é composto por inúmeras partes e o sapato tem de protegê-lo e imitar o seu movimento tendo apenas poucas partes principais. Além disso, o pé tem uma conexão direta com o bem-estar do restante do corpo e, por isso, o ajuste e o conforto são considerações importantes no design de sapatos.

O pé humano é considerado uma das estruturas biomecânicas do corpo humano com maior complexidade e, graças às suas características, proporciona ao corpo humano uma base estável que confere de forma eficiente não só o suporte e equilíbrio numa fase de apoio, como também uma estabilidade adequada durante o processo da marcha. (TABUAS, 2011)

O esqueleto do pé segundo Dangelo e Fattini (2006) é constituído por vinte e seis ossos, e dividido em três partes: o tarso, metatarso e falanges. O tarso é composto pelos ossos: tálus, calcâneo, navicular, cubóide e os três cuneiformes. O metatarso por cinco ossos longos. E as quatorze falanges formam os dedos do pé, sendo que cada um é composto por três falanges, exceto o hálux, conhecido popularmente como dedão, que possui apenas duas falanges maiores que as restantes dos outros dedos.

\footnotetext{
As necessidades de um projeto para suporte de uma carga estática em comparação com as de um aparelho para carga dinâmica são enormemente diferentes; ainda assim, o pé humano deve suprir essas duas demandas. Para tornar a questão ainda mais difícil, calçados estilizados ou mal ajustados forçam o pé a funcionar em posições anormais. Ao longo do curso de uma vida, tais fatores têm um efeito drástico na arquitetura e alinhamento dos ossos, das articulações e da estrutura dos tecidos moles circunjacentes. As alterações resultantes aumentam a chance de lesões agudas crônicas e por uso abusivo. (GALLO ET. AL, 2001 P. 361)
}

Gomes Filho (2010) alerta que a ergonomia no design de calçado é de fundamental importância, enfatizando os aspectos da correta utilização dos dados antropométricos disponíveis, bem como a modelagem em função dos ajustes necessários na definição dos tamanhos dos calçados. Bozano e Oliveira (2008) afirmam que a ergonomia pode contribuir na resolução de 
problemas encontrados em relação a conforto e saúde dos pés.

Para Guiel et. al (2006) com relação a funcionalidade do produto calçado, dois aspectos devem ser considerados: os movimentos do pé e o calce. $O$ autor também afirma que o conforto é obtido no artefato através do somatório das características: adequação da distribuição de peso do corpo; boa estabilidade com redução da pronação; boa capacidade de absorção de impactos; adequação dos componentes visando manter o peso do calçado em níveis de conforto; melhoria das propriedades térmicas da parte interna; boa flexibilidade e aderência adequada do calçado.

Choklat (2012) afirma que para garantir o conforto, algumas folgas-padrão devem ser criadas no interior do sapato. A elevação da biqueira, por exemplo, folga entre a base da ponta do sapato e o chão, é essencial e suaviza o movimento da caminhada. Outra folga importante é aquela entre a ponta dos dedos e o final do sapato, ela permite que o pé se movimente dentro do sapato durante o caminhar.

\section{Métodos}

O presente artigo foi desenvolvido utilizando como procedimento metodológico a Revisão Bibliográfica Sistemática (RBS) para selecionar os artigos relevantes para esta pesquisa, tendo como base o seguinte o problema: Quais os principais métodos sendo utilizados para análise ergonômica em calçados?

Antes de iniciar a RBS foram realizadas buscas exploratórias no banco de dados da Capes e duas dissertações foram encontradas que abordam o tema desta pesquisa e então serão complementares a mesma. Para realizar a revisão sistemática foi utilizada a plataforma Scopus, foram definidas as palavras chaves no idioma inglês e então gerado a seguinte string de busca: ("ergonomics" or "human factors") AND ("shoes" or "footwear") AND "design". Os critérios seleção de artigos publicados nos últimos cinco anos, leitura de títulos, abstracts, leitura diagonal (introdução, conclusão e métodos) e leitura completa do artigo também foram utilizados.

A busca foi então realizada no dia 31 de outubro de 2017 e gerou 58 documentos dentro dos critérios pré-estabelecidos. A primeira triagem foi executada utilizando título e palavras chaves, excluindo os artigos fora do contexto da pesquisa, resultado em 36 documentos. Posteriormente, os títulos e resumos de cada artigo foram analisados e descartados os que não se encaixavam a pesquisa devido ao tema, abordagem ou método. Foram assim selecionados seis artigos de acordo com sua relevância para a pesquisa. A seguir tabela com os documentos utilizados para o presente artigo, iniciando pelas dissertações e depois os artigos selecionados na RBS.

Tabela 1 - Artigos selecionados para análise

\begin{tabular}{llr}
\hline Título & Autores & Ano \\
\hline $\begin{array}{l}\text { Análise da pressão plantar de } \\
\text { mulheres com obesidade: } \\
\text { comparação de diferentes alturas de } \\
\text { calçados de salto }\end{array}$ & Ribeiro, M.S. & 2016 \\
$\begin{array}{l}\text { O conceito de inovação aplicado à } \\
\text { análise da relação produto/uso: o }\end{array}$ & Toledo, D.L. & 2017 \\
&
\end{tabular}


caso da sapatilha com ponta do balé clássico

Effects of indoor slippers on plantar pressure and lower limb EMG activity in older women

Modified track shoes and their effect on the EMG activity of calf muscles

Impacts of different types of insoles

on postural stability in older adults

Effects of heel cushioning elements in safety shoes on muscle-physiological parameters

Evaluation of slip resistance of safety footwear using novel equipment

inpact of different types of textile liners used in protective footwear on the subjective sensations of firefighters
Yick, K L

2016

Tse, L T

Lo, W T

$\mathrm{Ng}, \mathrm{S} P$

Yip, J

Greensword, M

2012

Aghazadeh, F

Al-Qaisi, S

Qu, X

2015

Huebner, A

2015

Schenk, P

Grassme, R

Anders, C

Bang, C H

2015

Kim, T.-G.

Kim, J.-S.

Irzmańska, E

2015

Fonte: elaborado pelos autores (2017)

\section{Resultado e Discussão}

Na sequência será apresentado uma análise dos objetivos, métodos e resultados de cada artigo selecionado através da revisão bibliográfica sistemática.

Ribeiro (2016) avaliou a pressão plantar de mulheres com obesidade, em duas situações: quando comparadas com um grupo de mulheres não obesas; e enquanto utilizam calçados de salto de alturas diferentes. Foram testados três calçados com alturas de saltos diferentes, como método o procedimento experimental consistiu na coleta dos picos de pressão plantar de cada voluntária, enquanto estavam paradas e andando, através do uso de uma palmilha sensorizada FScan ${ }^{\circledR}$ da Tekscan.

Foram avaliados e comparados: pico de pressão plantar dos dois grupos; pico de pressão plantar de cada calçado; percepção dos grupos quanto ao local de maior pico de pressão plantar; 
registro dos locais com maior pico de pressão plantar feito pelo programa de computador. Sendo que em todas as condições, o grupo de mulheres obesas registrou valores de picos de pressão mais elevados que do grupo de mulheres não obesas, e todas as avaliações indicaram o salto médio com o maior valor de pico de pressão. Outra avaliação subjetiva foi realizada utilizando o método de questionário, no qual ambos os grupos tiveram avaliações similares, diferente do programa, que apontou diferenças significativas entre as voluntárias obesas e as não obesas. (RIBEIRO 2016)

Toledo (2017) objetivou obter um diagnóstico e recomendações na conformação das sapatilhas de ponta do balé clássico. A coleta de dados foi realizada na Escola do Teatro Bolshoi no Brasil, com nove bailarinas. O método consistiu na mensuração dos pés e captação de imagens termográficas dos mesmos nos momentos pré e pós-utilização das sapatilhas de ponta e também foram realizadas entrevistas semiestruturadas com o grupo e com a fisioterapeuta da instituição. Os resultados obtidos demonstraram que com relação ao produto, o maior problema relatado ocorre na região das pontas dos pés e dedos, contudo ocorre de forma diferente para cada bailarina, o que sugeriu a necessidade de individualização das adequações utilizadas no calçado nos quesitos forma e material.

Yick et. al. (2016) avalia as propriedades físicas de dois típicos chinelos do tipo mule com abertura frontal e as mudanças na pressão plantar e atividade muscular dos membros inferiores das mulheres idosas ao usar estes calçados. Cinco experimentos de caminhada foram realizados por dez mulheres saudáveis. A velocidade de caminhada foi medida usando um portão de temporização automático que usa infravermelho. A pressão plantar foi medida com um sistema Pedar $^{\circledR}$ através de uma palmilha sensorizada, e o software Novel Multimask foi utilizado para coletar e analisar os dados. Os dados de EMG foram coletados de acordo com as diretrizes do projeto SENIAM (Hermens et al., 2000). E para finalizar um questionário que compreendeu seis itens em relação à percepção de conforto de diferentes aspectos dos calçado.

Os resultados indicam que, em comparação com os pés descalços, o desgaste de chinelos resulta em aumentos significativos na área de contato das regiões do meio do pé, o que leva à redistribuição da pressão plantar das cabeças metatarsianas 2-3 e do calcanhar lateral para as regiões do meio do pé. No entanto, não há diferença significativa na atividade muscular selecionada em todas as condições. Já o questionário apontou que, em geral, a maioria dos sujeitos preferem o chinelo mais macio, que é mais suave, mais leve e mais confortável do que o chinelo mais rígido.

Greensword et. al. (2012) avaliou os calçados com travas para esportistas. O experimento foi realizado com nove participantes que realizaram treinos de caminhada utilizando um calçado normal com travas e um modificado. A modificação consiste em adicionar um calcanhar removível à sola do sapato, a fim de reduzir a flexão do pé e nivelar adequadamente o pé para caminhar. 0 método utilizado foi a eletromiografia (EMG) para avaliar as atividades musculares, e um questionário onde os indivíduos avaliaram o desconforto em uma escala de 0-10. Os resultados mostram que o uso de calçados modificados resultou em uma redução de atividade EMG nos músculos, e as classificações de conforto aumentaram consideravelmente.

Qu (2015) examinou os efeitos de diferentes tipos de palmilhas sobre a estabilidade postural em idosos. O experimento contou com treze senescentes, incluiu uma sessão estática e uma sessão de caminhada, com quatro diferentes tipos de palmilhas. A velocidade média das 
séries temporais do centro da pressão obtidas a partir de uma plataforma de força foi utilizada para avaliar a estabilidade postural estática. A estabilidade postural dinâmica foi avaliada usando um sistema de captura de movimento de oito câmeras, utilizado para coletar os dados cinemáticos do corpo, enquanto os participantes caminhavam em uma esteira médica. Verificouse que a estabilidade postural estática não foi afetada por palmilhas, mas as palmilhas com abas melhoraram a estabilidade postural dinâmica, e a palmilha rígida estava associada a uma melhor estabilidade postural dinâmica em comparação com palmilhas macias.

Huebner et. al. (2015) avaliou as cargas de impacto associadas ao salto do calcanhar em sapatos de segurança. O método utilizado foi a eletromiografia superficial para analisar os músculos do tronco e das pernas dos 10 sujeitos do estudo, que caminharam por uma passarela enquanto usavam diferentes sapatos de segurança equipados com insertos que eram trocados de acordo com o peso corporal dos indivíduos. A utilização de sapatos com amortecimento otimizado conseguiram reduzir a tensão dos músculos, retardando a fadiga, e estendendo a proteção das articulações musculares.

Bang et. al. (2015) investiga a resistência ao deslizamento de calçados de segurança e objetiva determinar os principais fatores de projeto para melhorar a resistência ao deslizamento. Vinte e oito sapatos de segurança foram utilizados, em testes de fricção conduzidos sob três diferentes condições de contaminação, água, detergente e glicerol. Todos os testes de fricção foram realizados utilizando uma nova máquina de teste desenvolvida pela Agência de Segurança e Saúde Ocupacional da Coreia, que consiste em um suporte articulado desenvolvido para simular o movimento da marcha humana. Os resultados da medição de friç̧ão mostraram que calçados com solados em blocos extrudidos apresentam melhor resistência ao deslizamento, e a rigidez do solado também proporciona um maior coeficiente de atrito.

Irzmanska (2014) apresentou a avaliação ergonômica de botas de bombeiro com três tipos de revestimentos têxteis. O estudo foi conduzido em laboratório sob uma atmosfera normal com 45 bombeiros. As propriedades ergonômicas dos calçados de proteção e forros, sensações térmicas e de umidade experimentadas ao usar o calçado, foram avaliadas através do método questionário. O resultado confirmou que as propriedades ergonômicas do calçado de proteção usado no local de trabalho podem ser melhoradas pelo uso de componentes têxteis apropriados.

\subsection{Métodos identificados}

Diversos aspectos relacionados a ergonomia e conforto nos calçados podem ser analisados, como a pressão plantar, o equilíbrio estático ou dinâmico, o calce, a atividade muscular dos membros inferiores. Alguns artigos avaliaram os aspectos de apenas um determinado calçado enquanto outros utilizaram o mesmo método em diferentes tipos de calçados com a finalidade de comparar os dados, para auxiliar em um maior entendimento de quais características do calçado podem ser mais adequadas ergonomicamente.

Para avaliar a pressão plantar, a palmilha sensorizada se mostrou bastante eficiente tanto na pesquisa de Ribeiro (2016) quanto na de Yick et. al. (2016), mesmo usando palmilhas diferentes, foi possível chegar a resultados precisos e esclarecedores. Ambos os pesquisadores confrontaram os dados obtidos em diferentes tipos de calçados e Ribeiro (2026) também comparou os resultados dos calçados quando utilizados por dois grupos diferentes.

A Eletromiografia (EMG) estuda a função muscular por meio dos sinais elétricos emanados 
durante as contrações musculares, tornando possível acompanhar a atividade muscular através de eletrodos fixados na superfície da pele. Este método foi utilizado por Yick et. al. (2016), Greensword et. al. (2012) e Hubner et. al. (2015) para determinar entre os calçados especificados quais tinham menores índices de tensão nos músculos e consequentemente quais causavam menos fadiga e desgaste muscular. O EMG é um método tradicional já com diversas aplicações conhecidas e se mostrou eficaz para avaliar as atividades musculares na utilização de calçados.

Toledo (2017) utilizou o método de mensuração dos pés e captura de imagens termográficas objetivando avaliar qual a experiência das bailarinas ao usar a sapatilha de ponta. Os pontos de captura de imagens termográficas foram estabelecidos de acordo uma entrevista realizada previamente com as usuárias, o resultado apontou quais os pontos no pé em que o sapato restringe a circulação sanguínea e com maior tempo de uso retira a sensibilidade da ponta dos dedos. O método se revelou interessante para a pesquisa em questão, e pode ser útil em outros estudos sobre conforto em calçados de uso cotidiano.

Para analisar a estabilidade postural relacionada a palmilhas Qu (2015) utilizou uma plataforma de força para avaliar a estabilidade postural estática e obteve o resultado de que a mesma não foi afetada pelo uso das diferentes palmilhas. Para avaliação da estabilidade postural dinâmica o autor coletou os dados cinéticos do corpo através de um sistema de captura de movimentos que apontou diferenças significativas entre as palmilhas.

Bang et. al. (2015) utilizou uma máquina de teste que simula o movimento da marcha humana para investigar a resistência de calçados de segurança ao deslizamento. O método teve eficaz aplicação para comparação do solado de diversos tipos de calçados, porém não foi realizado nenhum outro tipo de teste diretamente com seres humanos.

Toledo (2017) utilizou o método entrevista, e os autores Ribeiro (2016), Yick et. al. (2016) e Greensword et. al. (2012) utilizaram questionário para complementar a pesquisa e comparar com os dados obtidos através de outros métodos. Irzmanka foi o único pesquisador que utilizou somente questionário como método para avaliar as propriedades ergonômicas, sensações térmicas e de umidade no calçado em questão, o que torna o resultado subjetivo. $O$ artigo aponta a relação existente do material do forro com o desconforto, porém não explica de forma objetiva sua influência, visto que considerou apenas a percepção do usuário, a inclusão de outros métodos poderiam trazer uma conclusão mais precisa.

Sabendo que não existe melhor ou um único método, é necessário buscar qual o mais adequado para a pesquisa em questão. Os artigos com análises que utilizaram mais de um método chegaram a resultados mais precisos e interessantes, confrontar e comparar dados de métodos diferentes pode enriquecer a pesquisa.

\section{Considerações finais}

Com o propósito de organizar e sintetizar em um único artigo alguns dos principais métodos que podem ser utilizados para avaliar ergonomicamente diferentes aspectos de um calçado, este trabalho alcançou seu objetivo. Através de uma revisão bibliográfica sistemática foi possível identificar e analisar artigos com relevância para o tema proposto e compará-los com duas dissertações selecionadas previamente.

Devido à complexidade de avaliar diferentes aspectos referentes à ergonomia de um calçado foram identificados variados métodos nos artigos, e a maioria deles utilizaram um 
conjunto de dois ou mais métodos que se complementaram para resultados mais precisos.

Portanto, é essencial ter conhecimento sobre os diferentes tipos de métodos e suas variadas aplicabilidades, para iniciar futuras pesquisas com maior embasamento de qual possa ser o método mais adequado. Ressalta-se também que o uso de mais de um método traz maior confiabilidade e relevância para os resultados obtidos. A utilização destes métodos na análise ergonômica de calçados é importante para a melhoria e adequação deste artefato ao usuário, resultando no aumento da condição de conforto e conseqüentemente reduzindo a incidência de problemas causados pelo uso de calçados inadequados.

\section{Referências}

ANSELMO, T. K. Parâmetros para o desenvolvimento de sapatilhas femininas de uso diário com ênfase no conforto térmico e percepção da usuária. Disponível em <http://www.tede.udesc.br/tde_busca/arquivo.php?codArquivo=3837> Acesso em: 12 de jan. 2016.

BANG, C. H.; KIM, T. -G.; KIM, J.-S. Evaluation of slip resistance of safety footwear using novel equipment. Disponível em < https://www.scopus.com/inward/record.uri?eid=2-s2.084962917562\&doi=10.1016\%2Fj.apergo.2016.03.018\&partnerID=40\&md5=74e9c112e96c9d4788 9390b5224fa224> Acesso em: 31 de out. 2017.

CHOKLAT, A. Design de Sapatos. São Paulo: Editora Senac São Paulo, 2012.

DANGELO, J. G.; FATTINI, C. A. Anatomia básica dos sistemas orgânicos: com a descrição dos ossos, junturas, músculos, vasos e nervos. São Paulo: Editora Atheneu, 2006.

GOMES FILHO, J. Ergonomia do objeto: sistema técnico de leitura ergonômica. São Paulo: Escrituras Editora, 2010.

GREENSWORD, M.; AGHAZADEH, F.; AL-QUAISI, S. Modified track shoes and their effect on the EMG activity of calf muscles. Disponível em < https://www.scopus.com/inward/record.uri?eid=2s2.0-84859871088\&doi=10.3233\%2FWOR-2012-0382-

1763\&partnerID=40\&md5=15a107a475a7d8de6e7aa53cb3a7222a> Acesso em: 31 de out. 2017.

GUIEL, A. V.; BERWANGER, E. G.; QUEIROZ, J. L.; SCHMIDT, M. R.; HAISSER, M. Dossiê técnico: desenvolvimento do produto em calçados. Disponível em < http://www.sbrt.ibict.br/dossietecnico/downloadsDT/OA==>. Acesso em 16 de mar. 2016.

HUEBNER, A.; SCHENK, P.; GRASSME, R.; ANDERS, C. Effects of heel cushioning elements in safety shoes on muscle-physiological parameters. Disponível em $<$ https://www.scopus.com/inward/record.uri?eid=2-s2.084939208088\&doi=10.1016\%2Fj.ergon.2015.01.009\&partnerlD=40\&md5=9cab171b20504552412 ffad1f85e9505> Acesso em: 31 de out. 2017.

IRZMANSKA, E. The impact of different types of textile liners used in protective footwear on the subjective sensations of firefighters. Disponivel em $<$ https://www.scopus.com/inward/record.uri?eid=2-s2.084919600466\&doi=10.1016\%2Fj.apergo.2014.08.013\&partner ID=40\&md5=0bc57fbf1d217c05649 2f7d9eb259f48> Acesso em: 31 de out. 2017.

QU, X. Impacts of different types of insoles on postural stability in older adults. Disponível em < 


\section{Artigo Completo}

https://www.scopus.com/inward/record.uri?eid=2-s2.0-

84908170955\&doi=10.1016\%2Fj.apergo.2014.06.005\&partnerID=40\&md5=2d7a22f34aa71f298b4 d6a4696c6ed54 > Acesso em: 31 de out. 2017.

RIBEIRO, M. S. Análise da pressão plantar de mulheres com obesidade: comparação de diferentes alturas de calçados de salto. Dissertação (Mestrado em Design) - Programa de Pós Graduação em Design, UDESC - SC, Florianópolis, 2016.

SANTOS, Flavio Anthero Nunes Vianna dos. Nota de aula disciplina: Design, Método e Fatores Humanos - CURSO DE PÓS-GRADUAÇÃO EM DESIGN PPGD - Mestrado Métodos para fatores humanos - Florianópolis, UDESC - Universidade do Estado de Santa Catarina, 06 de setembro de 2017.

TABUAS, C. F. D. Análise da Pressão Plantar para fins de Diagnóstico. Disponível em < https://web.fe.up.pt/ tavares/downloads/.../MEB_TP_CTabuas.pdf>. Acesso em: 20 de jan. 2016.

TOLEDO, L. T. O conceito de inovação aplicado à análise da relação produto/uso: o caso da sapatilha com ponta do balé clássico. Dissertação (Mestrado em Design) - Programa de Pós Graduação em Design, UFSC - SC, Florianópolis, 2017. 